\title{
EXTENSIONS OF NICE BASES ON ULM SUBGROUPS OF PRIMARY ABELIAN GROUPS WITH TOTALLY PROJECTIVE QUOTIENTS
}

\author{
PETER DANCHEV AND PATRICK KEEF
}

\begin{abstract}
Suppose $G$ is an abelian $p$-group, $\alpha$ is an ordinal and $G / p^{\alpha} G$ is totally projective. We show that $G$ has a nice basis if and only if $p^{\alpha} G$ has a nice basis. This extends results of Danchev in Bull. Malaysian Math. Sci. Soc. (2010), Bull. Allah. Math. Soc. (2011) and An. St. Univ. Ovidius Constanta (2012), as well as joint work with Keef in Rocky Mount. J. Math. (2011).
\end{abstract}

\section{INTRODUCTION}

Throughout this paper, the term "group" will mean an additively written abelian $p$-group, where $p$ is a fixed prime. All terminology not explicitly defined herein will agree with [6].

If $\alpha$ is ordinal and $G$ is a group, then $p^{\alpha} G$ is defined inductively as follows: $p^{0} G=G$, and if $\alpha>0$, then $p^{\alpha} G=\cap_{\beta<\alpha} p\left(p^{\beta} G\right)$. This subgroup is also called $\alpha$-th Ulm subgroup of $G$.

Suppose $G$ is a group and $\alpha$ is an ordinal such that the quotient $G / p^{\alpha} G$ is totally projective. A problem of some interest in abelian group theory is to describe important group properties which hold for $G$ if and only if they hold for $p^{\alpha} G$. This type of investigation was initiated by Hill in [7], and was further developed by several other authors - see, for example, [4] and [5].

Recall that the group $G$ possesses a nice basis if it is the countable ascending union of nice subgroups that are direct sums of cyclic groups; if these nice subgroups are bounded, we will say that the group is equipped with a bounded nice basis. For nice bases, theorems of the present type were established in [1], [2], [3] and [5]. In [1] and [5], the first author proved that $G$ has a nice basis if and only if $p^{\omega} G$ has a nice basis provided that $G / p^{\omega} G$ is totally projective (i.e., a direct sum of cyclic groups). Later on, this was

2010 Mathematics Subject Classification. 20K10.

Key words and phrases. Primary Abelian groups, nice bases, totally projective groups, Ulm subgroups, Ulm factors. 
extended in [2] to ordinals $\alpha<\omega^{2}$ and, furthermore, generalized in [3] to all ordinals $\alpha \leq \Omega$ (notice that in both cases the factor-group $G / p^{\alpha} G$ is a direct sum of countable groups). Analogous results were obtained for bounded nice bases.

The purpose of this note is to generalize these results to an arbitrary ordinal $\alpha$ by showing that whenever the quotient group $G / p^{\alpha} G$ is totally projective (i.e., simply presented), then $G$ has a (bounded) nice basis if and only if $p^{\alpha} G$ has a (bounded) nice basis - see Theorem 2.1.

Finally, we ask whether this characterizes the totally projective groups of length $\alpha$. To that end, we will say a group $A$ of length $\alpha$ has the the nice basis $\alpha$-extension property if for all groups $G$ with $G / p^{\alpha} G \cong A$, then $G$ has a nice basis if and only $p^{\alpha} G$ has a nice basis (for $\alpha=\omega$, in [5] this was called the nice basis extension property). So Theorem 2.1 states that all totally projective groups of length $\alpha$ have the nice basis $\alpha$-extension property. We ask whether the converse of Theorem 2.1 holds, i.e., are all groups with the nice basis $\alpha$-extension property actually totally projective? For $\alpha=\omega$, a partial answer was given in [5].

\section{THE MAIN RESUlT}

We begin with an explicit definition of our main term.

Definition. A nice basis for a group $G$ is an ascending sequence of nice subgroups $\left\{N_{i}\right\}_{i<\omega}$ such that each $N_{i}$ is a direct sums of cyclic subgroups and $G=\cup_{i<\omega} N_{i}$. If each $N_{i}$ is bounded, then $\left\{N_{i}\right\}_{i<\omega}$ is a bounded nice basis.

We now establish our main result which answers Problem 1 from [3].

Theorem 2.1. Suppose $\alpha$ is an ordinal and $G$ is a group such that $G / p^{\alpha} G$ is totally projective. Then $G$ has a nice basis if and only if $p^{\alpha} G$ has a nice basis.

Proof. Necessity. In 79(g) of [6] it was observed that when $N$ is nice in $G$, then $N \cap p^{\alpha} G$ is nice in $p^{\alpha} G$. This readily implies that if $\left\{N_{i}\right\}_{i<\omega}$ is a nice basis for $G$, then $\left\{N_{i} \cap p^{\alpha} G\right\}_{i<\omega}$ is a nice basis for $p^{\alpha} G$, as desired.

Sufficiency. Let $\left\{M_{n}\right\}_{n<\omega}$ be a nice basis for $p^{\alpha} G$.

Let $B$ be a basic subgroup of $p^{\alpha} G$. It follows from a consideration of Ulm functions that there is a simply presented group $H$ such that $p^{\alpha} H=B$ and $H / p^{\alpha} H \cong G / p^{\alpha} G$ (see, for example, Theorems 76.1 and 83.6 of [6]). Now, if we let $K=\left(H \oplus p^{\alpha} G\right) /\{(x,-x): x \in B\}$, then we can think of $H$ and $p^{\alpha} G$ as subgroups of $K$ such that $H+p^{\alpha} G=K$ and $H \cap p^{\alpha} G=B=p^{\alpha} H$. Since $K / B \cong\left(H / p^{\alpha} H\right) \oplus\left(p^{\alpha} G / B\right)$, where the first term is $p^{\alpha}$-bounded and the second term is divisible, it follow that $p^{\alpha} K=p^{\alpha} G$. Therefore, since

$$
K / p^{\alpha} K=\left(H+p^{\alpha} G\right) / p^{\alpha} G \cong H /\left(H \cap p^{\alpha} G\right)=H / p^{\alpha} H \cong G / p^{\alpha} G
$$


is simply presented, it follows that $K \cong G$ (see, for instance, Theorem 83.4 of [6]). So we may assume that $K=G$, so that $H \subseteq G$.

Let $\mathcal{S}$ be the generators in some simple presentation of $H$. It follows that

$$
\left\{b+p^{\alpha} G: b \in S \text { and } h t_{G}(b)<\alpha\right\}
$$

are the generators in a simple presentation of $G / p^{\alpha} G \cong H / p^{\alpha} H$.

If $n<\omega$, let

$$
\mathcal{S}_{n}=\left\{b \in \mathcal{S}: p^{n} b \in M_{n} \text { and }\langle b\rangle \cap p^{\alpha} H=\langle b\rangle \cap M_{n}\right\} .
$$

It is easy to check that if $x \in \mathcal{S}_{n}$, then $p x \in \mathcal{S}_{n}$. Set $T_{n}=\left\langle\mathcal{S}_{n}\right\rangle \subseteq G$; clearly $p^{n} T_{n} \subseteq M_{n}$. Finally, let $N_{n}=M_{n}+T_{n}$, so that $p^{n} N_{n} \subseteq M_{n}$, as well.

We shall verify that the following four conditions hold:

(1) $N_{n}$ is a direct sum of cyclic groups.

(2) $N_{n} \cap p^{\alpha} G=M_{n}$, so that it is nice in $p^{\alpha} G$.

(3) $\left(N_{n}+p^{\alpha} G\right) / p^{\alpha} G=\left\langle b+p^{\alpha} G: b \in \mathcal{S}_{n}\right\rangle$ is nice in $G / p^{\alpha} G$.

(4) $\mathcal{S}=\cup_{n<\omega} \mathcal{S}_{n}$.

To verify (1), note that since $M_{n}$ is a direct sum of cyclics, the same can be said of $p^{n} N_{n} \subseteq M_{n}$; which also implies that $N_{n}$ is a direct sum of cyclics.

As for (2), the modular law from [6] assures that $N_{n} \cap p^{\alpha} G=M_{n}+\left(T_{n} \cap\right.$ $\left.p^{\alpha} G\right)$; so we need to show that $T_{n} \cap p^{\alpha} G \subseteq M_{n}$.

The definition of $\mathcal{S}_{n}$ implies that $\mathcal{S}_{n} \cap p^{\alpha} H=\mathcal{S}_{n} \cap M_{n} \subseteq M_{n}$. Therefore, $T_{n} \cap p^{\alpha} G=\left(T_{n} \cap H\right) \cap p^{\alpha} G=T_{n} \cap\left(H \cap p^{\alpha} G\right)=T_{n} \cap p^{\alpha} H=\left\langle\mathcal{S}_{n} \cap p^{\alpha} H\right\rangle \subseteq M_{n}$, which proves $(2)$.

Concerning point (3), the equality follows from the definitions, and the statement about niceness from 83(f) in [6].

In checking (4), suppose $b \in \mathcal{S}$. Let $b+p^{\alpha} G$ have order $p^{k}$ in $G / p^{\alpha} G \cong$ $H / p^{\alpha} H$. Choose $n \geq k$ such that $p^{k} b \in M_{n}$. It follows that $\langle b\rangle \cap p^{\alpha} H=$ $\left\langle p^{k} b\right\rangle=\langle b\rangle \cap M_{n}$, which means that $b \in \mathcal{S}$.

So, we are done verifying (1)-(4).

Note that statements (2) and (3) imply that $N_{n}$ is nice in $G$ (see, for example, [6]), and by (1) it is a direct sum of cyclics. Moreover, since $M_{n} \subseteq N_{n}$ and $p^{\alpha} G=\cup_{n<\omega} M_{n}$, condition (4) implies that $G=\cup_{n<\omega} N_{n}$. So $\left\{N_{n}\right\}_{n<\omega}$ forms a nice basis for $G$, as required.

The same argument works for groups with bounded nice basis as well. We therefore state without proof the following analogue of Theorem 2.1.

Corollary 2.2. Suppose $\alpha$ is an ordinal and $G$ is a group such that $G / p^{\alpha} G$ is totally projective. Then $G$ has a bounded nice basis if and only if $p^{\alpha} G$ has a bounded nice basis.

We can restate Theorem 2.1 as follows: 
Corollary 2.3. If $\alpha$ is an ordinal and $A$ is a totally projective group of length $\alpha$, then $A$ has the nice basis $\alpha$-extension property.

We close the article with the following question of interest, which asks if the converse of the last statement holds.

Problem. If $\alpha$ is an ordinal, is it true that every group with the nice basis $\alpha$-extension property is totally projective? That is, suppose $A$ is a group of length $\alpha$ such that whenever $G$ is a group such that $A \cong G / p^{\alpha} G$ and $p^{\alpha} G$ each have a nice basis, then $G$ itself has a nice basis. Can we conclude that $A$ is totally projective?

\section{REFERENCES}

[1] P. V. Danchev, Nice bases for mixed and torsion-free abelian groups, Bull. Malays. Math. Sci. Soc., 33 (3) (2010), 393-403.

[2] P. V. Danchev, Extending nice bases on Ulm subgroups of abelian p-groups, Bull. Allah. Math. Soc., 26 (2) (2011), 225-228.

[3] P. V. Danchev, An extension of nice bases on Ulm subgroups of primary abelian groups, An. St. Univ. Ovidius Constanta, 20 (3) (2012), 33-36.

[4] P. V. Danchev and B. Goldsmith, On socle-regularity and some notions of transitivity for abelian p-groups, J. Comm. Algebra, 3 (3) (2011), 301-319.

[5] P. V. Danchev and P. W. Keef, Nice bases and thickness in primary abelian groups, Rocky Mount. J. Math., 41 (4) (2011), 1127-1149.

[6] L. Fuchs, Infinite Abelian Groups, Volumes I and II, Acad. Press, New York and London, 1970 and 1973.

[7] P. D. Hill, On transitive and fully transitive primary groups, Proc. Amer. Math. Soc., 22 (2) (1969), 414-417.

(Received: June 8, 2012)

(Revised: October 23, 2012)
Peter Danchev

Mathematical Department

Plovdiv State University, Plovdiv 4000

Bulgaria

pvdanchev@yahoo.com

peter.danchev@yahoo.com

Patrick Keef

Mathematical Department

Whitman College

Walla Walla, WA 99362

U. S. A.

keef@whitman.edu 